\title{
BMC Geriatrics reviewer acknowledgement 2014
}

Giulia Mangiameli

\section{Contributing reviewers}

The editors of BMC Geriatrics would like to thank all our reviewers who have contributed to the journal in Volume 14 (2014).

Wilco Achterberg

Netherlands

Francisco Acurcio

Brazil

Kristina Akesson

Sweden

Theresa Allain

Malawi

Fred Andersen

Norway

Melissa K. Andrew

Canada

Hidenori Arai

Japan

Glenn Arendts

Australia

Joshua Armstrong

Canada

Benoit Arsenault

Netherlands

Erik Augustson

USA

Xue Bai Hong

Kong

Gillian Barry

UK

Peter Bartlett

UK
Stephane Baudry

Belgium

Marla Beauchamp

USA

Clemens Becker

Germany

Giuseppe Bellelli

Italy

Joel Belmin

France

Melissa Benton

USA

Rainer Beurskens

Germany

Kamaldeep Bhui

UK

Mark Bicket

USA

Ross Black

Australia

Florian Bleibler

Germany

Thomas Bodenheimer

USA

Froukje Boersma

Netherlands

Erin Bouldin

USA
Elisabeth Boulton

UK

Susan Bowles

Canada

Michael Brach

Germany

Elizabeth Breeze

UK

Emily Brogan

Australia

Rebecca Brown

USA

Ana-Maria Buga

Germany

Frances Bunn

UK

Matthew Bush

USA

Bianca Maria Buurman

Netherlands

Jeff Caird

Canada

Philip Calder

UK

Simone Caljouw

Netherlands

Michele Callisaya

Australia

Correspondence: giulia.mangiameli@biomedcentral.com

BioMed Central, Floor 6, 236 Gray's Inn Road, London WC1X 8HB, UK 


\begin{tabular}{|c|c|c|}
\hline Marcela Carrasco & Shameka Cody-Humphrey & Lars Donath \\
\hline Chile & USA & Switzerland \\
\hline Calogero Caruso & Mauro Colombo & Wendy Duggleby \\
\hline Italy & Italy & Canada \\
\hline Robin Casten & Simon Conroy & Eamonn Eeles \\
\hline USA & UK & Australia \\
\hline Carlo Cervellati & Rachel Cooper & Thorlene Egerton \\
\hline Italy & UK & Norway \\
\hline Ding-Cheng Chan & Bernard Cortet & Maha El Gaafary \\
\hline Taiwan & France & Egypt \\
\hline Yoon-Seok Chang & Michael Crary & Grahame Elder \\
\hline South Korea & USA & Australia \\
\hline Satabdi Chatterje & Marilyn Cree & Coralie English \\
\hline USA & Canada & Australia \\
\hline Pui Hing Chau & Nathan Davies & Mark Fagan \\
\hline Hong Kong & UK & Norway \\
\hline Huey-Tzy Chen & Jennifer Davis & Rui Felgueiras \\
\hline Taiwan & Canada & Portugal \\
\hline Huashuai Chen & Piers Dawes & Steffen Fieuws \\
\hline USA & UK & Netherlands \\
\hline Chung-Hwan Chen & Eling D. De Bruin & Joseph Finkelstein \\
\hline Taiwan & Switzerland & USA \\
\hline Liang-Kung Chen & Stefanie De Buyser & Trine Elisabeth Finnes \\
\hline Taiwan & Belgium & Norway \\
\hline Shuai Chen & Leen De Coninck & Thomas Fischer \\
\hline USA & Belgium & Germany \\
\hline Yu-Chun Chen & Lieven De Maesschalck & George Fitchett \\
\hline Taiwan & Belgium & USA \\
\hline Sheung-Tak Cheng & Cesar De Oliveira & Johan Flamaing \\
\hline Hong Kong & UK & Belgium \\
\hline Karen Siu Lan Cheung & Francesca De Terlizzi & Leon Flicker \\
\hline Hong Kong & Italy & Australia \\
\hline Mei Sian Chong & Marjolein De Vugt & Scott Forbes \\
\hline Singapore & Netherlands & Canada \\
\hline Kee-Lee Chou & Anja Declercq & Kristian Steen Frederiksen \\
\hline China & Belgium & Denmark \\
\hline Vivienne Chuter & Kim Delbaere & Ellen Freiberger \\
\hline Australia & Australia & Germany \\
\hline Arrigo Fg Cicero & Liesbet Demarré & Michael Freitag \\
\hline Italy & Belgium & Germany \\
\hline Andrew Claus & Mario Di Napoli & Yoshinori Fujiwara \\
\hline Australia & Italy & Japan \\
\hline Antonio Coca & Martin Dichter & Yoshiharu Fukuda \\
\hline Spain & Germany & Japan \\
\hline
\end{tabular}




\begin{tabular}{|c|c|c|}
\hline Carlo Gabelli & Man Guo & Ruth Jepson \\
\hline Italy & USA & UK \\
\hline Michelle Gagnon & Thora Hafsteinsdottir & Antony Johansen \\
\hline Canada & Netherlands & UK \\
\hline Josep Garre-Olmo & Klaus Hager & Lena Johansson \\
\hline Spain & Germany & Sweden \\
\hline Nick Gebruers & Ruud Halfens & Becca Jordre \\
\hline Belgium & Netherlands & USA \\
\hline Suzanne Geerlings & Christine Hartmann & Anthony Jorm \\
\hline Netherlands & USA & Australia \\
\hline Rebecca Genoe & Yvonne Heerkens & Olivia Kada \\
\hline Canada & Netherlands & Austria \\
\hline Clarissa Giebel & Stephan Heinzel & Pekka Kannus \\
\hline UK & Germany & Finland \\
\hline Timothy Girard & Jorunn Helbostad & Gertrudis I.J.M. Kempen \\
\hline USA & Norway & Netherlands \\
\hline Andrea Giusti & Francois Herrmann & Marie-Jeanne Kergoat \\
\hline Italy & Switzerland & Canada \\
\hline Danijela Gnjidic & Mira Hidajat & Ngaire Kerse \\
\hline Australia & USA & New Zealand \\
\hline Fernando Goglia & Andy Hau Yan Ho & Rahul Khanna \\
\hline Italy & Hong Kong & USA \\
\hline Adam L Gordon & Lee Hooper & Mijin Kim \\
\hline UK & UK & South Korea \\
\hline Urs Granacher & Frances Horgan & Naruki Kitano \\
\hline Germany & Ireland & Japan \\
\hline William B. Grant & Fei-Yuan Hsiao & Taro Kojima \\
\hline USA & Taiwan & Japan \\
\hline Amanda Grenier & Kuangshi Huang & Daniel Kopf \\
\hline Canada & China & Germany \\
\hline Meredith Gresham & Paulette Hunter & Goran Koracevic \\
\hline Australia & Canada & Serbia \\
\hline Yves Gschwind & Bettina Husebo & Rumi Kozakai \\
\hline Australia & Norway & Japan \\
\hline Danan Gu & Valeria Isella & Reto W. Kressig \\
\hline USA & Italy & Switzerland \\
\hline Antonio Guaita & Tatsuro Ishizaki & Morten Tange Kristensen \\
\hline Italy & Japan & Denmark \\
\hline Valeri Guajardo & Susan Jaglal & Edeltraut Kröger \\
\hline Brazil & Canada & Netherlands \\
\hline Maelenn Guerchet & Mary Janevic & Vilai Kuptniratsaikul \\
\hline UK & USA & Thailand \\
\hline Jason Guertin & Barbara Jefferis & Alexander Kurz \\
\hline Canada & UK & Germany \\
\hline
\end{tabular}


Linda Lam

Hong Kong

Sarah Lamb

UK

Claudine Lamoth

Netherlands

Carole-Lynne Le Navenec

Canada

Jean Yves Le Reste

France

\section{Sandra Lefort}

Canada

Anja Leist

Luxembourg

Melanie Lesinski

Germany

Joan Leung

Australia

Melanie Li

Taiwan

Yi Li

China

Tianyuan Li

Hong Kong

Jae-Young Lim

South Korea

Ulrich Lindemann

Germany

Richard Lindley

Australia

Tsan-Hon Liou

Taiwan

Benjamin Liptzin

USA

Melanie Logue

USA

Carl Lombard

South Africa

Vivian W. Q. Lou

Hong Kong

Lee-Fay Low

Australia

Lisa Low

Hong Kong
Nan Lu

China

Elena Lucchi

Italy

Terry Lum

Hong Kong

Duncan Macfarlane

Hong Kong

Shylie Mackintosh

Australia

Davide Malatesta

Switzerland

Daniel Malone

USA

Alayne Markland

USA

Ana Isabel Marques

Portugal

Manuel Martin-Carrasco

Spain

Nicolás Martínez-Velilla

Spain

Emanuele Marzetti

Italy

Roy Mathew

USA

Colleen Maxwell

Canada

Cathal Mccrory

Ireland

Jane Mccusker

Canada

Katherine S Mcgilton

Canada

Patrizia Mecocci

Italy

Graydon Meneilly

Canada

Gabriele Meyer

Germany

Laura Middleton

Canada

Amanda Miller Amberber

Australia
Veronica Mocanu

Romania

Roberto Monastero

Italy

Kirsten Moore

Australia

Nicholas Moore

France

Rodrigo Moreira

Brazil

Mario Luca Morieri

Italy

Cristiano Moura

Canada

Martin Mueller

Germany

Judy Muller-Delp

USA

Nanette Mutrie

UK

Koutatsu Nagai

Japan

Alicia Nelson

USA

Karin Neufeld

USA

Louisa Ng

Australia

Tuan Nguyen

Australia

Anna Nieboer

Netherlands

Akitoshi Nishishita

Japan

Shwe Zin Nyunt

Singapore

Christiane Oedekoven

UK

Nelly Oelke

Canada

Yoshiro Okubo

Japan

Marcel Olde Rikkert

Netherlands 
Jacilyn Olson

USA

Desmond O'Neill

Ireland

Bregje D Onwuteaka-Philipsen

Netherlands

Carlos Orces

USA

Matthew Parsons

New Zealand

Sahdia Parveen

UK

Anita Patel

UK

Eyvind Paulssen

Norway

Juan D. Pedrera-Zamorano

Spain

Carlo Pedrolli

Italy

Nancye May Peel

Australia

Geeske Peeters

Australia

Eugen-Bogdan Petcu

Australia

Genevieve Pham Kanter

USA

Evelien Pijpers

Netherlands

Giulio Pioli

Italy

Jenny Ploeg

Canada

Kristina Radinovic

Serbia

Parvin Rahnama

Iran

Kilian Rapp

Germany

Michael Rapp

Germany

Irene Maeve Rea

UK
Bjorn Rosengren

Sweden

Renzo Rozzini

Italy

Alessandro Rubinacci

Italy

Jorge Ruivo

Portugal

Tom Russ

UK

David Russell

USA

Liv Wergeland Sorbye

Norway

Mahshid Saghazadeh

Japan

Zhu Sai Nan

China

Fabio Salvi

Italy

Raluca Elena Sandu Vintilescu

Romania

Gill Sarre

UK

Kai-Uwe Saum

Germany

Ingmar Schäfer

Germany

Lori Schindel Martin

Canada

Daniel Schoene

Australia

Birgitte Jjm Schoenmakers

Belgium

Nadja Schott

Germany

Rene Schwendimann

Switzerland

Michael Schwenk

USA

David Scott

Australia

Carlo Serrati

Italy
Hyehyung Shin

South Korea

Shoji Shinkai

Japan

Hiromi Shiraishi

Japan

Joanie Sims-Gould

Canada

Sarianna Sipilä

Finland

Martin Smalbrugge

Netherlands

Alex Smithson

Spain

Rafael Solana

Spain

Stanislaw Solnik

USA

Yuki Soma

Japan

Yuting Song

USA

Vincent Staggs

USA

Els Steeman

Belgium

Magnus Stenhagen

Sweden

Joanna Stewart

New Zealand

Junko Sugama

Japan

Caroline Sutcliffe

UK

Michele Talley

USA

Nanako Tamiya

Japan

Rajesh Tampi

USA

Cara Tannenbaum

Canada

Kristin Taraldsen Norway 
Der-Cherng Tarng

Taiwan

Hans Thulesius

Sweden

Chris Todd

UK

Christophe Trivalle

France

Taishi Tsuji

Finland

Kenji Tsunoda

Japan

Anthony Tuckett

Australia

Maarit Katariina Valtonen

Finland

Marian A.E. Van Bokhorst - De

Van Der Schueren

Netherlands

Lieve Van Den Block

Belgium

Marlise Van Eersel

Netherlands

\section{Paula Van Wyk}

Canada

David Vance

USA

Massimo Venturelli

Italy

Hilde Verbeek

Netherlands

Davide Liborio Vetrano

Italy

Renuka Visvanathan

Australia

Angela Vivanti

Australia
Stefano Volpato

Italy

Wolfgang Von Renteln-Kruse

Germany

Ake B. Wahlin

Sweden

Jingjy Wang

Taiwan

Pei-Ning Wang

UK

Christine Wann-Hansson

Sweden

Katherine Ward

USA

Rachel Ward

USA

Eiichi Watanabe

Japan

Joseph Wherton

UK

Julie Whitney

UK

Birgitt Wiese

Germany

Jacek M. Witkowski

Poland

Peter Wolf

Switzerland

Karin Wolf-Ostermann

Germany

Martin Wolkewitz

Germany

Bettina Wollesen

Germany

Gloria Wong

Hong Kong
Kuangnan Xiong

USA

Dongjuan Xu

USA

Fang Yang

China

Yen Kuang Yang

Taiwan

Charles W. Yates

USA

Dannii Yeung

Hong Kong

Abebaw, Mengistu Yohannes

UK

Jiyeong Yoon

Japan

Jieun Yoon

Japan

Andrea Zammit

USA

Laura Zannini

Italy

Astrid Zech

Germany

Fengyu Zhang

USA

Xin Zhang

China

Li Zhang

China

Quan Zhou

China

Haiyan Zhu

USA

Giovanni Zuliani

Italy 